\title{
Slip-length measurement of confined air flow using dynamic atomic force microscopy
}

\author{
Abdelhamid Maali \\ Centre de Physique Moléculaire Optique et Hertzienne, Université Bordeaux 1, F-33405 Talence, France \\ Bharat Bhushan* \\ Nanoprobe Laboratory for Bio- \& Nanotechnology and Biomimetics, Ohio State University, Columbus, Ohio 43210-1142, USA
}

(Received 30 November 2007; revised manuscript received 24 July 2008; published 26 August 2008)

\begin{abstract}
We present an experimental measurement of the slip length of air flow close to solid surfaces using an atomic force microscope (AFM) in dynamic mode. The air was confined between a glass surface and a spherical glass particle glued to an AFM cantilever. The Knudsen number was varied continuously over three decades by varying the distance between the two surfaces. Our results show that the effect of confining the air is purely dissipative. The data are described by an isothermal Maxwell slip-boundary condition, and the measured slip-length value was $118 \mathrm{~nm}$.
\end{abstract}

DOI: 10.1103/PhysRevE.78.027302

PACS number(s): 47.10.ad, 47.45.Gx, 83.50.Lh, 83.50.Rp

The fluid flow close to solid surfaces has been studied in detail during the last century [1-13]. The velocity profile of a thin fluid film exhibits a phenomenon known as a slip, which means that the fluid velocity near the solid surface is not equal to the velocity of the solid surface. The existence of slip was first predicted by Maxwell for gas flow close to a wall [1]. Slip is important for ultrathin films, especially when the fluid is confined. Due to the recent advances in microand nanoelectromechanical systems (MEMS and NEMS) (e.g., micro- and nanofluidics) and in computer-storage devices, the available space for the gas is reduced, and the laws governing the flow at this scale need to be understood $[4,6-9,12,13]$. To describe the gas flow, a Knudsen number $K_{D}$ has been introduced, and it is related to the molecular mean free path of the gas, $\lambda$, and the characteristic length of the flow domain, $D$, as $K_{D}=\lambda / D$. When the Knudsen number is on the order of 0.01 or larger, rarefaction of the gas has to be taken into account and slip occurs on the surfaces [5-9]. The slip velocity is given by Maxwell's slip conditions for an isothermal wall,

$$
V_{s}=\left.b \frac{\partial V}{\partial z}\right|_{z=0}=\left.\left(\frac{2-\sigma}{\sigma}\right) \lambda \frac{\partial V}{\partial z}\right|_{z=0},
$$

where $b$ is the slip length, $\sigma$ is the accommodation coefficient that is a measure of the fraction of the gas molecules that are reflected diffusively from the solid surface, $V$ and $V_{s}$ are the tangential velocity in the fluid flow and its value at the surface, respectively, and $z$ is the vertical axis, orthogonal to the solid surfaces. The slip length is defined as the negative vertical intercept of the velocity profile.

Our research is motivated by recent work on the measurement of liquid slip on solid surfaces using an atomic force microscope (AFM) in contact mode [14-17] and surface force apparatus (SFA) $[18,19]$. In the AFM experiments, the viscous force acting on a spherical surface has been measured during approach at a constant velocity to a solid sur-

\footnotetext{
*Corresponding author. FAX: +1-614-292-0325.

face. For hydrophobic surfaces, the measured viscous force was always smaller than the calculated one assuming a noslip-boundary condition. By adding to the expression of the calculated force a function that takes into account the liquid slip [20], researchers extract the slip-length value from their data. These techniques were applied successfully to different surfaces and different liquids. However, such apparatuses are not suitable for the study of gas flow because the induced viscous force is very low, due to the viscosity of the gas being lower by two orders of magnitude, and is difficult to measure accurately. In the dynamic force apparatus, the liquid slip on hydrophobic surfaces has been measured within a resolution of $1 \mathrm{~nm}$ [19]. But the stiffness of the cantilever that is used is too high (on the order of several thousand $\mathrm{N} / \mathrm{m}$ ) to be able to measure the forces due to confined gases, and the quality factor is low (on the order of 30) with a low sensitivity to probe the hydrodynamic interaction of the confined gas.

In this paper, we used an AFM in dynamic mode to investigate the confined air flow close to solid surfaces. The air was confined between a glass surface and a spherical glass particle glued to an AFM cantilever. Due to the high-quality factor of the cantilever far from the solid surface, the additional damping induced by the hydrodynamic interaction during the confining of the gas can be probed accurately by just measuring the variation of the amplitude and the phase of the cantilever as the sphere approaches the solid surface.

For a cantilever oscillating with a small amplitude compared to the range of the interaction length $(\lambda, b)$, the interaction force acting on the cantilever can be linearized and has two contributions; one is a conservative term $\left(-k_{H} z\right)$ due to the elastic compression of the film, and the other is a dissipative term $\left(-\gamma_{H} \dot{z}\right)$ due to viscous damping during the squeezing; $k_{H}$ and $\gamma_{H}$ are hydrodynamic interaction stiffness and damping coefficients, respectively, and $z$ is the instantaneous position of the cantilever. The motion of the cantilever is then described by $m^{*} \ddot{z}+\left(\gamma_{0}+\gamma_{H}\right) \dot{z}+\left(k_{l}+k_{H}\right) z$ $=F_{0} \exp (j \omega t)$ and the driving force 

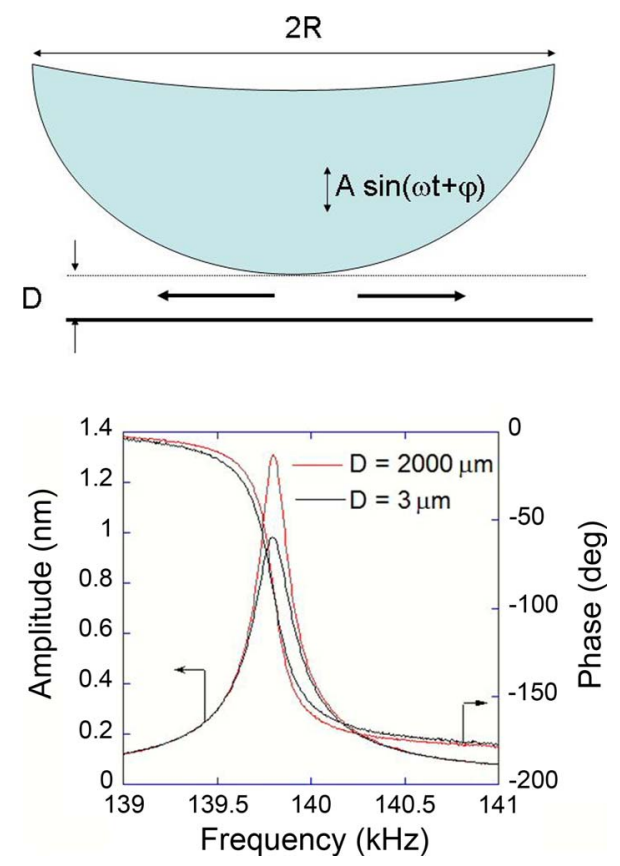

FIG. 1. (Color online) Amplitude and phase spectra of the cantilever obtained at two different positions from the glass surface.

$$
F_{0}=\frac{k_{l} A_{0}}{Q},
$$

where $m^{*}$ is the effective mass of the cantilever, $\gamma_{0}$ is the bulk viscous damping far from the surface and is related to the quality factor $Q$ and the resonance frequency $\omega_{0}$ via the equation $Q=\frac{m^{*} \omega_{0}}{\gamma_{0}}, k_{l}$ is the cantilever stiffness, and $A_{0}$ is the amplitude of the oscillation far away from the interaction region. The steady-state solution $z=A \exp j(\omega t+\varphi)$ of the above equation gives the stiffness and damping coefficients [21]:

$$
k_{H}=k_{l}\left[\frac{A_{0} \cos (\varphi)}{A Q}-1+\frac{\omega^{2}}{\omega_{0}^{2}}\right]
$$

and

$$
1+\gamma_{H} / \gamma_{0}=-\frac{\omega_{0} A_{0}}{\omega A} \sin (\varphi)
$$

where $A$ and $\varphi$ are the measured amplitude and phase of the oscillation, respectively, and $\omega$ is the driving frequency of the cantilever.

A spherical glass particle (diameter of $34 \mu \mathrm{m}$ ), purchased from Duke Corporation, was glued on the cantilever-tip side of silicon tapping cantilever (resonance frequency of $332 \mathrm{kHz}$ and $Q$ of 420) using an epoxy glue. The substrate used was a flat glass surface. The substrate surface roughness measured using a 10-nm-radius silicon tip cantilever over 4 $\times 4 \mu \mathrm{m}^{2}$ was $1.2 \mathrm{~nm}$ and peak-to-valley distance of less than $4 \mathrm{~nm}$. The experiments were performed using a commercial AFM (NanoScope III-extended Multimode, Veeco Instruments) at ambient laboratory conditions. The vibrating amplitude and phase are measured using a lock-in amplifier
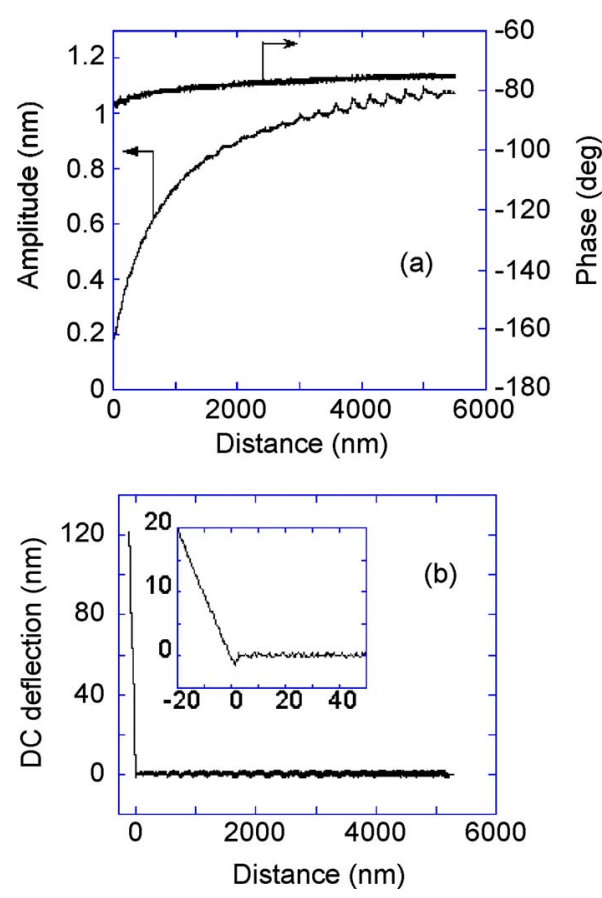

FIG. 2. (Color online) (a) The measured amplitude and phase of the cantilever as the glued sphere approaches the glass substrate (the vibration frequency is $139.8 \mathrm{kHz}$, the amplitude far from the interaction is $1.3 \mathrm{~nm}$, and the gas pressure is $1 \mathrm{~atm})$. The modulation on the amplitude signal is due to the interferences due to multiple reflections on both surfaces (cantilever and substrate) of the laser light used to measure the cantilever motion. (b) The dc deflection of the cantilever that allows one to determine the zero distance (contact position of the two surfaces). The inset shows a close-up of the dc-deflection curve at small distance.

(Signal Recovery Model 7280). Figure 1 shows the amplitude and phase spectra of the cantilever far from the glass surface $(2000 \mu \mathrm{m})$ and at a distance of $3 \mu \mathrm{m}$. By approaching the surface the resonance frequency remains unchanged and only the amplitude and phase vary. To study the interaction due to the confined air, instead of measuring the amplitude and phase spectra as a function of the distance, we use an amplitude modulation (AM) mode. We fix the operating frequency and record the vibration amplitude and phase versus the distance. The distance between the substrate and the mean position of the tip is reduced at a rate $0.572 \mu \mathrm{m} / \mathrm{s}$ until the two surfaces touch each other, and then they are moved apart with the same rate.

Figure 2 shows the measured amplitude and phase and the $\mathrm{dc}$ deflection of the cantilever as the distance between the glass sphere and the glass substrate is reduced. The cantilever was vibrated close to the resonance frequency $(20 \mathrm{~Hz}$ below the resonance frequency) with an amplitude of $1.3 \mathrm{~nm}$ far from the surface. For each sphere-substrate surface gap, the amplitude and phase of the cantilever and the dc deflection of the cantilever were measured. The dc deflection allows one to determine the contact position of the two surfaces with an accuracy better than $1 \mathrm{~nm}$ [Fig. 2(b)]. Note in Fig. 2(a) that due to the squeezed viscous air between the sphere and the surface, the amplitude starts to reduce at a distance far away from the surface. To get the amplitude very 


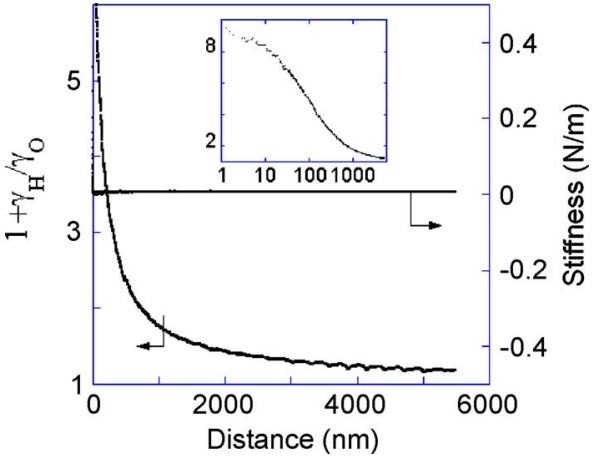

FIG. 3. (Color online) The normalized hydrodynamic damping $1+\gamma_{H} / \gamma_{0}$ and the hydrodynamic interaction stiffness $\left(k_{H}\right)$ versus the distance $(D)$ for the sphere approaching the glass surface. The inset shows $1+\gamma_{H} / \gamma_{0}$ versus the distance on semilogarithmic plot.

far from the surface $(2000 \mu \mathrm{m})$, the two surfaces were displaced using a stepper motor. From the amplitude and phase data, we calculated the interaction stiffness and damping using Eqs. (3) and (4). Figure 3 shows the damping $1+\gamma_{H} / \gamma_{0}$ and the stiffness $k_{H}$ as the distance $D$ was diminished. The damping increases as the sphere approaches the glass surface due to the increase of the viscous drag force; however, the stiffness is unchanged and is equal to zero. From this observation, we infer that there is no elastic part in the force and the interaction is purely dissipative. Note here that the compression effect in our situation of a sphere vibrating close to solid surfaces is lower than the usual studied case of air squeezed between two parallel disks [13]. In our case, the squeezing is only important for the surface area of the sphere close to the glass surface; the rest of the sphere does not contribute to the squeezing.

For a gas confined in a volume $v$ with a fixed number of molecules, the variation of the volume has to be compensated by a variation of the pressure, $\frac{\Delta p}{p} \cong \frac{\Delta v}{v}$. One may expect that as the sphere vibrates it induces a volume change which has to be compensated for by the pressure change, which results in an elastic force that will act on the sphere glued to the cantilever. Our experiments show that it is not the case and the interaction is purely dissipative (no elastic part). During the vibration of the sphere, the gas has the time to escape from the confining region. For the usual cases encountered in MEMS and NEMS, the surfaces are parallel with a gap that does not depend on the lateral direction parallel to the surfaces [13]. For those cases, the gas compression is characterized by a squeeze number that is calculated after linearization of the Reynolds equation [13,22,23], $\sigma=\frac{12 \eta \omega L^{2}}{p_{a} D^{2}}$, where $L$ is the typical length of the surfaces, $p_{a t}$ is the atmospheric pressure, and $\eta$ is the viscosity, which is pressure independent for an ideal gas. For a small gap, the squeeze number in the case of parallel surfaces can be very large. In our case, the gap has a radial dependence, which is given in the lubrication approximation by $h(r) \approx D+\frac{r^{2}}{2 R}$, where $D$ is the closest distance between the sphere and the substrate. Using the same argument, we calculate the gas squeeze number that is equal to $\sigma_{\text {sphere }}=\frac{4 \eta \omega R}{p_{a} D}$. Introducing our experimental values into the above expression, we see that even at a distance as small as $D=100 \mathrm{~nm}, \sigma_{\text {sphere }}=0.13<1$, and thus the compression effect is negligible.

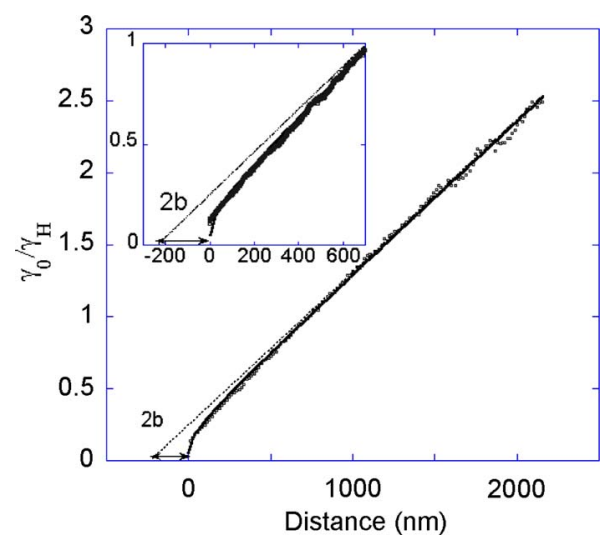

FIG. 4. (Color online) The inverse of the normalized hydrodynamic damping, $\gamma_{0} / \gamma_{H}$, versus the distances between the sphere and the glass surface. The solid line is the fit curve using theoretical expression [Eq. (6)]. The dashed line corresponds to the linear extrapolation of the signal it intersects the distance axis at position $2 b=236 \mathrm{~nm}$.

In the case of the no-slip-boundary conditions, the hydrodynamic force opposing the motion of the sphere at distance $D$ from the substrate surface is given by the Taylor equations

$$
F_{H}=\gamma_{H} \dot{z}=\frac{6 \pi \eta R^{2}}{D} \dot{z}
$$

where $R$ is the sphere radius, $D$ is the gap between the surfaces, $\eta$ is the viscosity of the air, and $\dot{z}$ is the instantaneous velocity of the sphere. The inverse of the normalized hydrodynamic damping is given as $\frac{\gamma_{0}}{\gamma_{H}}=\frac{\gamma_{0} D}{6 \pi \eta R^{2}}$. Figure 4 presents the inverse of the normalized hydrodynamic damping $\gamma_{0} / \gamma_{H}$ obtained from the data presented in Fig. 3. We observe that the data curve does not pass through the origin, which means that the air flow close to the solid surface presents a slip like liquid flow close to a hydrophobic surface. To take into account the slip effect, we introduce a correction function into the hydrodynamic damping [20]:

$$
\gamma_{0} / \gamma_{H}=\frac{\gamma_{0}}{6 \pi \eta R^{2}} \frac{D}{f(D)}
$$

where

$$
f(D)=\frac{D}{3 b}\left[\left(1+\frac{D}{6 b}\right) \ln \left(1+\frac{6 b}{D}\right)-1\right] .
$$

Here, the viscosity is taken to be constant and the gas is allowed to slip on both surfaces with the same slip-length value $b$. From the fit, we get a slip length of $118 \pm 10 \mathrm{~nm}$. Uncertainty in the measurements comes from the surface roughness of the glass as well as the noise present at large distances. At a large distance, the damping is small, which is responsible for the scatter. By fitting the data at various distances, we get a scatter of $\pm 10 \mathrm{~nm}$. For the air at atmospheric pressure and ambient temperature, the mean free path is $67 \mathrm{~nm}$ [5], and thus the accommodation coefficient that corresponds to the measured slip length, based on Eq. (1), is about $\sigma=0.72$. The accommodation coefficient was reported to be in the range of $0.5-1$ for different gases on different 
surfaces $[7,9]$. Actually the most used techniques are based on the measurement of mass flow through a microchannel $[7,9]$. For a given microchannel, the gap is fixed, and the parameter which is varied is the difference of the pressure between the inlet and the outlet of the microchannel. Thus the slip is studied for a given gap value. Here the gap is varied continuously, and thus the gas flow behavior was studied as a function of the gap (the corresponding Knudsen number varies from 0.01 to 10 ).

The Taylor equation combined with the slip function (both derived from the Navier-Stokes equation) describes well our results with good accuracy. Recent results on gas transport through a carbon nanotubule [9] demonstrated also that the experiments agree well with the continuous equation. This study demonstrates that the hydrodynamic continuous models have to be investigated thoroughly before disregarding them. Our experiments open the way for more study of confined gas at the nanometer scale.

To conclude, we have presented a measurement of the vibrations of a spherical glass particle close to a smooth solid surface. As the distance between the sphere and the surface is reduced, the damping increases due to the confined gas between the surfaces. The Knudsen number was varied continuously over three decades by varying the distance between the two surfaces. Our results show that the effect of flow on the cantilever is purely dissipative, which means that the fluid is not compressed even at high Knudsen number. Furthermore the damping is described by the Taylor equation, in which we introduce a correction factor that takes into account the slip of the gas on the surfaces. The extracted slip length is $118 \mathrm{~nm}$, which corresponds to an accommodation coefficient of $\sigma=0.72$ for air on the glass. The atomic force microscope in dynamic mode been successfully used to measure the slip length in a confined gas.
[1] J. C. Maxwell, Philos. Trans. R. Soc. London 170, 231 (1867).

[2] R. A. Millikan, Phys. Rev. 21, 217 (1923).

[3] D. L. Morris, L. Hannon, and A. L. Garcia, Phys. Rev. A 46, 5279 (1992).

[4] T. Veijola, H. Kuisma, J. Lahdenperä, and T. Ryhänen, Sens. Actuators, A 48, 239 (1995).

[5] G. A. Bird, Molecular Gas Dynamics and the Direct Simulation of Gas Flows (Oxford University Press, Oxford, 1996).

[6] B. Bhushan, Tribology and Mechanics of Magnetic Storage Devices, 2nd ed. (Springer-Verlag, New York, 1996).

[7] E. B. Arkilic, K. E. Breuer, and M. A. Schmidt, J. Fluid Mech. 29, 437 (2001).

[8] D. A. Lockerby, J. M. Reese, D. R. Emerson, and R. W. Barber, Phys. Rev. E 70, 017303 (2004).

[9] S. M. Cooper, B. A. Cruden, and M. Meyyapan, Nano Lett. 4, 377 (2004).

[10] B-Y. Cao, M. Chen, and Z-Y. Guo, Appl. Phys. Lett. 86, 091905 (2005).

[11] E. Lauga, M. P. Brenner, and H. A. Stone, in Handbook of Experimental Fluid Dynamics edited by J. Foss, C. Tropea, and A. Yarin (Springer, New York, 2005).

[12] B. Bhushan, Springer Handbook of Nanotechnology, 2nd ed. (Springer-Verlag, Heidelberg, 2007).

[13] M. Bao and H. Yang, Sens. Actuators, A 136, 3 (2007).

[14] J. W. G. Tyrrell and P. Attard, Phys. Rev. Lett. 87, 176104 (2001).

[15] E. Bonaccurso, M. Kappl, and H.-J. Butt, Phys. Rev. Lett. 88, 076103 (2002).

[16] E. Bonaccurso, H.-J. Butt, and V. S. J. Carig, Phys. Rev. Lett. 90, 144501 (2003).

[17] C. Neto, D. R. Evans, E. Bonaccurso, H.-J. Butt, and V. S. J. Craig, Rep. Prog. Phys. 68, 2859 (2005).

[18] Y. Zhu and S. Granick, Phys. Rev. Lett. 88, 106102 (2002).

[19] C. Cottin-Bizonne, B. Cross, A. Steinberger, and E. Charlaix, Phys. Rev. Lett. 94, 056102 (2005).

[20] O. I. Vinogradova, Langmuir 11, 2213 (1995).

[21] J. P. Aimé, R. Boisgard, L. Nony, and G. Couturier, J. Chem. Phys. 114, 4945 (2001).

[22] W. A. Gross, Gas Film Lubrication (Wiley, New York, 1962).

[23] W. E. Langlois, Q. Appl. Math. 20(2), 131 (1962). 